\title{
Nonlinear excitations, stability inversions, and dissipative dynamics in quasi-one-dimensional polariton condensates
}

\author{
J. Cuevas, ${ }^{1}$ A. S. Rodrigues,${ }^{2}$ R. Carretero-González, ${ }^{3}$ P. G. Kevrekidis, ${ }^{4}$ and D. J. Frantzeskakis ${ }^{5}$ \\ ${ }^{1}$ Grupo de Física No Lineal, Departamento de Física Aplicada I. Escuela Politécnica Superior, Universidad de Sevilla, \\ C/ Virgen de Árica, 7, ES-41011 Sevilla, Spain \\ ${ }^{2}$ Departamento de Física/CFP, Faculdade de Ciências, Universidade do Porto, R. Campo Alegre 687, PT-4169-007 Porto, Portugal \\ ${ }^{3}$ Nonlinear Dynamical Systems Group, Department of Mathematics and Statistics, and Computational Science Research Center, \\ San Diego State University, San Diego California, 92182-7720, USA \\ ${ }^{4}$ Department of Mathematics and Statistics, University of Massachusetts, Amherst, Massachusetts 01003-4515, USA \\ ${ }^{5}$ Department of Physics, University of Athens, Panepistimiopolis, Zografos, Athens GR-157 84, Greece
}

(Received 9 February 2011; published 30 June 2011)

\begin{abstract}
We study the existence, stability, and dynamics of the ground state and nonlinear excitations, in the form of dark solitons, for a quasi-one-dimensional polariton condensate in the presence of nonresonant pumping and nonlinear damping. We find a series of remarkable features that can be directly contrasted to the case of the typically energy-conserving ultracold alkali-atom Bose-Einstein condensates. For some sizable parameter ranges, the nodeless ("ground") state becomes unstable toward the formation of stable nonlinear single- or multi-dark-soliton excitations. It is also observed that for suitable parametric choices, the instability of single dark solitons can nucleate multi-dark-soliton states. Also, for other parametric regions, stable asymmetric sawtooth-like solutions exist. These are shown to emerge through a symmetry-breaking bifurcation from bubble-like solutions that we also explore. We also consider the dragging of a defect through the condensate and the interference of two initially separated condensates, both of which are capable of nucleating dark multisoliton dynamical states.
\end{abstract}

DOI: $10.1103 /$ PhysRevB.83.245140

PACS number(s): 03.75.Lm, 05.45.Yv, 71.36.+c

\section{INTRODUCTION}

An important recent development that has spurted a new direction for the physics of Bose-Einstein condensation has been the observation of exciton-polariton Bose-Einstein condensates (BECs) in semiconductor microcavities ${ }^{1}$ at much higher temperatures than ultracold atomic BECs. ${ }^{2}$ In the setting of exciton-polariton BECs, the excitons (i.e., bound electron-hole particles), when confined, develop a strong coupling with light, forming exciton-photon mixed quasiparticles known as polaritons. ${ }^{3}$ The polariton radiative lifetime is of the order of $1-10 \mathrm{ps}$, which is typically shorter than all typical relaxation times in the system. ${ }^{4}$ The much lighter mass, by about four orders of magnitudes in comparison to the excitons of such exciton-polaritons (due to their photonic component), renders them excellent candidates for solid-state BEC. Yet, this short lifetime, also inherited by their photonic component, does not allow thermalization, hence yielding a (typically) nonequilibrium setting (thermalization may ensue as a result of strong polariton-polariton interaction caused by their excitonic component ${ }^{5}$ ). Here, the external pumping from a reservoir of excitons counterbalances the loss of polaritons due to the above mentioned decay mechanism. Nevertheless, many key features of the superfluid character of exciton-polariton BECs have been established, including the flow without scattering (analog of the flow without friction), ${ }^{6}$ the existence of vortices ${ }^{7}$ (see also Ref. 8 for vortex dipole dynamics), the collective dynamics, ${ }^{9}$ as well as remarkable applications such as spin switches ${ }^{10}$ and light-emitting diodes ${ }^{11}$ operating even at near-room temperatures.

The pumping and damping mechanisms associated with polaritons enable the formulation of different types of models. One of these, proposed in Refs. 12 and 13, suggests the use of a single partial differential equation (PDE) for the polariton condensate incorporating the above mentioned lossgain mechanisms. This model features a localized (within a pumping region) gain and a nonlinear saturating loss of polaritons; these are the fundamental differences of this setting from the standard PDE mean-field model, namely the Gross-Pitaevskii equation (GPE), used in the physics of atomic BECs. ${ }^{2,14}$ In another class of models, which has been proposed in Refs. 15-17, the polaritons are coupled to the evolution of the exciton population; such models also display nonlinear diffusive spatial dynamics of the excitons.

In this work, our aim is to study the quasi-one-dimensional (1D) dynamics of nonresonantly excited polariton BECs and to illustrate their fundamental differences from alkali-atom condensates. Here we should note that, in most cases, the polariton condensates considered so far (also experimentally) have been intrinsically two-dimensional (2D). ${ }^{18}$ Nevertheless, a tight confinement along a transverse direction (i.e., using highly anisotropic variants of the traditional harmonic traps), may render the polariton condensate effectively $1 \mathrm{D} .{ }^{19}$ Furthermore, it is relevant to mention that quasi-1D trapping of the polariton BEC using a thin microwire to "guide" the condensate, has already been demonstrated in recent experiments. ${ }^{20} \mathrm{~A}$ broader perspective for our considerations is that understanding the nonlinear dynamics and pertinent phenomenology in the 1D setting, may pave the way toward subsequently generalizing relevant considerations to the $2 \mathrm{D}$ case. The key phenomena that are reported herein are the following: a wide parametric interval of destabilization of the fundamental nodeless ("ground") state of polariton BECs, a partial stability within this interval of excited states in the form of dark solitons, the spontaneous production of higher excited (multi-soliton) states from lower ones or even from nodeless states, the emergence 
of asymmetric sawtooth states through the symmetry-breaking of symmetric bubble ones, the production of dark-soliton trains by dragging of a defect through the polariton BEC (cf. the recent relevant 2D experimental results in Ref. 21), and finally the formation of long-lived multiple-dark-soliton states through the interference of two separated polariton condensates, in analogy with the atomic BEC case. ${ }^{22}$

The paper is organized as follows. In Sec. II we describe our model and setup, providing also a brief description of our methods. Section III is devoted to our detailed numerical investigations, and Sec. IV concludes our work, including suggestions for future studies.

\section{MODEL SETUP}

In our analysis below, we consider the modified complex Gross-Pitaevskii model developed in Refs. 12 and 13 suitably reduced to one spatial dimension (see the recent experiment of Ref. 20, where a thin microwire was used to implement such a quasi-1D setting),

$$
i \partial_{t} \psi=\left\{-\partial_{x}^{2}+x^{2}+|\psi|^{2}+i\left[\chi(x)-\sigma|\psi|^{2}\right]\right\} \psi .
$$

In the above model, $\psi$ denotes the polariton wave function, and $x^{2}$ represents an effectively $1 \mathrm{D}$ external harmonic potential (notice that the transverse direction, perpendicular to $x$, corresponds to the tight-trapping axis mentioned above). This external potential can be created by lithographical masks imprinted on the microcavity wafers, ${ }^{23}$ or by mechanically pressing the microcavity wafer. ${ }^{24}$ The differences of Eq. (1) from the standard GPE appearing in the physics of atomic BECs can be traced to the presence of (i) the spatially dependent gain term of the form

$$
\chi(x)=\alpha \Theta\left(x_{m}-|x|\right),
$$

where $\Theta$ is the step function generating a symmetric pumping spot of "radius" $x_{m}$ and strength $\alpha$ for the gain, and (ii) the nonlinear saturation loss term of strength $\sigma$. Estimates of the relevant physical time and space scales, as well as physically relevant parameter values, are given in Ref. 12. We should mention that although our results below are given in the context of Eq. (1), we have ensured that a similar phenomenology arises in the model of Refs. 15-17 for suitable parametric choices; in other words, the phenomenology that is reported in this work is generically relevant to $1 \mathrm{D}$ polariton BECs independently of model specifics. We also note in passing that Ginzburg-Landau-type models, similar to the one of Eq. (1) - i.e., including a localized gain term (but, in most cases, without the external harmonic potential)_were recently studied in the context of nonlinear optics ${ }^{25}$ and in the physics of magnon condensates. ${ }^{26}$

In what follows, we will consider the stationary solutions of the quasi-1D model at hand, in the form $\psi(x, t)=$ $\psi_{0}(x) \exp (-i \mu t)$, where $\mu$ is the dimensionless chemical potential, and the stationary state $\psi_{0}(x)$ is governed by the following ordinary differential equation:

$$
\mu \psi_{0}=\left\{-\frac{d^{2}}{d x^{2}}+x^{2}+\left|\psi_{0}\right|^{2}+i\left[\chi(x)-\sigma\left|\psi_{0}\right|^{2}\right]\right\} \psi_{0} .
$$

Importantly, the additional condition

$$
\int d x\left[\chi(x)-\sigma\left|\psi_{0}\right|^{2}\right]\left|\psi_{0}\right|^{2}=0
$$

needs to be enforced as a population-balance constraint. This self-consistently selects the particular value of the chemical potential once the other parameters (i.e., $\alpha, \sigma$, and $x_{m}$ ) are fixed. This is why some of our graphs of the solution branches below will feature $\mu$ as a function of other solution parameters, such as $x_{m}$. We note in passing the significant difference of this trait from the Hamiltonian of the atomic BEC case, where there exist monoparametric families of solutions as a function of $\mu$. Once stationary solutions of the differential-algebraic system of Eqs. (3) and (4) are identified, their linear stability is considered by means of a Bogolyubov-de Gennes analysis. Specifically, considering small perturbations [of order $\mathrm{O}(\delta)$, with $0<\delta \ll 1]$ of the stationary solutions, we substitute the ansatz

$$
\psi(x, t)=e^{-i \mu t}\left\{\psi_{0}(x)+\delta\left[a(x) e^{i \omega t}+b^{*}(x) e^{-i \omega^{*} t}\right]\right\}
$$

into Eq. (1), and then solve the ensuing [to $\mathrm{O}(\delta)$ ] eigenvalue problem:

$$
\omega\left(\begin{array}{l}
a(x) \\
b(x)
\end{array}\right)=\left(\begin{array}{cc}
L_{1} & L_{2} \\
-L_{2}^{*} & -L_{1}^{*}
\end{array}\right)\left(\begin{array}{l}
a(x) \\
b(x)
\end{array}\right)
$$

for the eigenvalue $\omega$ and associated eigenvector $[a(x), b(x)]^{T}$. Here, $L_{1}$ and $L_{2}$ are the following operators:

$$
\begin{aligned}
& L_{1}=-\mu-\frac{d^{2}}{d x^{2}}+x^{2}+2(1-i \sigma)\left|\psi_{0}\right|^{2}+i \chi(x), \\
& L_{2}=(1-i \sigma) \psi_{0}^{2} .
\end{aligned}
$$

Once the stationary solutions are found to be linearly unstable (i.e., $\operatorname{Im}\{\omega\} \neq 0$ ), then the dynamics of the respective instabilities is monitored through direct numerical simulations of Eq. (1).

In addition, in what follows, we consider dynamical scenarios under which nonlinear excitations, such as single or multiple dark solitons ${ }^{14}$ can arise in the context of polariton BECs. Such excited states have been extensively studied in the context of atomic BECs, ${ }^{27}$ while they have been amply considered in recent experimental investigations in this context. $^{22,28,29}$ Furthermore, motivated by relevant studies in atomic BECs, ${ }^{30,31}$ as well as recent experiments in polariton condensates, ${ }^{6,21,32}$ we consider the nucleation of dark solitons by a moving defect, modeled by a strongly-localized potential; the latter is assumed to be produced by a narrow laser beam of Gaussian shape, namely,

$$
V_{\mathrm{def}}=V_{0} \exp \left[-(x-v t)^{2} / \epsilon^{2}\right],
$$

where $V_{0}, v$, and $\epsilon$ represent the amplitude, speed, and width of the potential, respectively. We consider the fixed-point solution of the modified GPE, Eq. (1), in the presence of this second defect potential (in addition to the harmonic trap $x^{2}$ ) at the center of the trap. Then, we evolve the system in time starting from this solution, dragging the defect through the system. This is similar in spirit to the recent experiments of Refs. 6 and 21 (and to the recent theoretical investigation of Ref. 33). Finally, we study an alternative proposal for dark-soliton nucleation, akin to the interference experiments conducted for atomic 
BECs in Ref. 22, whereby a central potential barrier separating two polariton clouds is lifted allowing the two clouds to interfere; we show that this process leads to the production of persistent dark solitons as well.

\section{NUMERICAL RESULTS}

We hereby explore the existence, stability, and dynamical properties of the nodeless cloud (NC) as well as of excited states exhibiting a single node, namely dark solitons (DSs). The above mentioned states (NC and DS) are the most fundamental nonlinear states of the system, whose profilesfor different parameter combinations - are displayed in Fig. 1. In particular, the left panel of Fig. 1 depicts the NC and DS profiles for a constant saturation coefficient $\sigma=0.35$ and constant pumping spot strength $\alpha=2$, and a varying radius of the pumping spot $x_{m}$. We observe that, for given values of $\alpha$ and $\sigma$, there is a critical value of $x_{m}$ above which the shapes of the NCs and DSs remain unchanged. In the example depicted in the left panel of Fig. 1, the profiles for $x_{m} \gtrsim 3$ are indistinguishable from each other (see profiles for $x_{m}=3$ and $x_{m}=5$ ). This effect is due to the fact that for large $x_{m}$, the pumping spot covers a larger portion than the saturated size of the cloud when loss and gain are balanced. This cloud size is the analog of the Thomas-Fermi radius for an atomic condensate. The saturation of the cloud size is equivalent to the saturation of the chemical potential $\mu$ as $x_{m}$ is increased, as shown below (see Figs. 4 and 5). In the right panel of Fig. 1 we depict the NC and DS profiles for a constant pumping spot radius $x_{m}=3$ and a varying pumping spot strength. Note that, in this case, the size of the cloud continuously expands (in amplitude and width) with increasing pumping spot strength.

We now proceed to provide a characterization of the existence and stability properties of the NC and DS profiles with respect to the various parameters at hand. In what follows, in order to offer a picture of the relevant parameter space, we have varied the gain parameters $\alpha$ and $x_{m}$ whereas the coefficient of the saturating nonlinear loss $\sigma=0.35$ has been

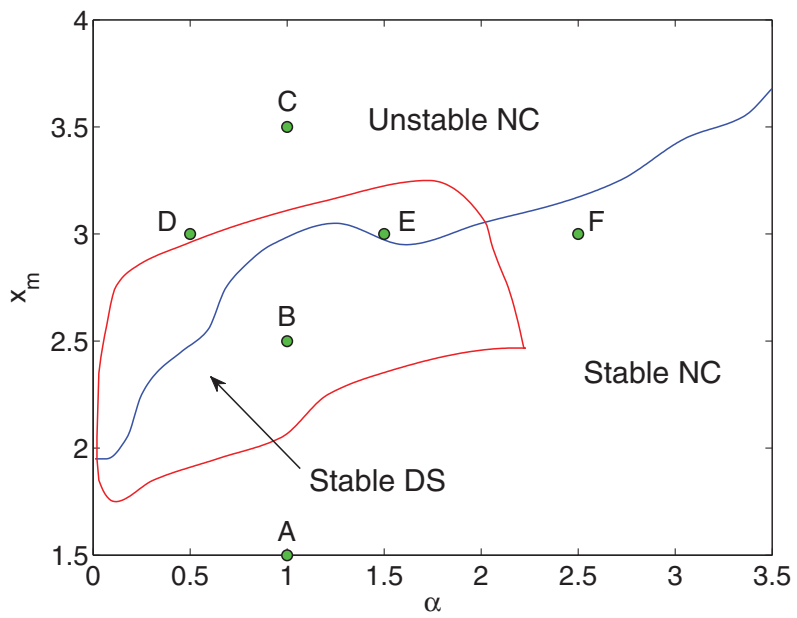

FIG. 2. (Color online) Stability domains of nodeless clouds (NC) and dark solitons (DSs) for $\sigma=0.35$ and $\alpha \leqslant 3.5$. Dark solitons are stable only in the area indicated by the arrow. The (green) circles indicate the parameter locations for the excitation spectra depicted in Fig. 3.

kept fixed. Figure 2 depicts the existence and stability domains in the $\left(\alpha, x_{m}\right)$-parameter plane with $\sigma=0.35$ fixed for both the NC and the single DS that can be found as (numerically exact up to a prescribed accuracy) fixed-point solutions of Eq. (3). The NC and DS configurations exist for all parameter combinations, as it is the case for atomic condensates.

Nevertheless, as far as the stability and dynamical properties of NC and DS states are concerned, we can observe fundamental differences between the pumped-damped polariton BECs and atomic BECs. In particular, the nodeless cloud (which was always stable in the Hamiltonian case of atomic $\mathrm{BECs}^{14}$ ) is now stable only below a critical value of the pumping-spot size $x_{m}$. On the other hand, also remarkably, even the single DS is stable only in a limited range, while it was always stable in quasi-1D harmonically trapped atomic BECs (see, e.g., Ref. 22 and references therein). Moreover, in a very unusual
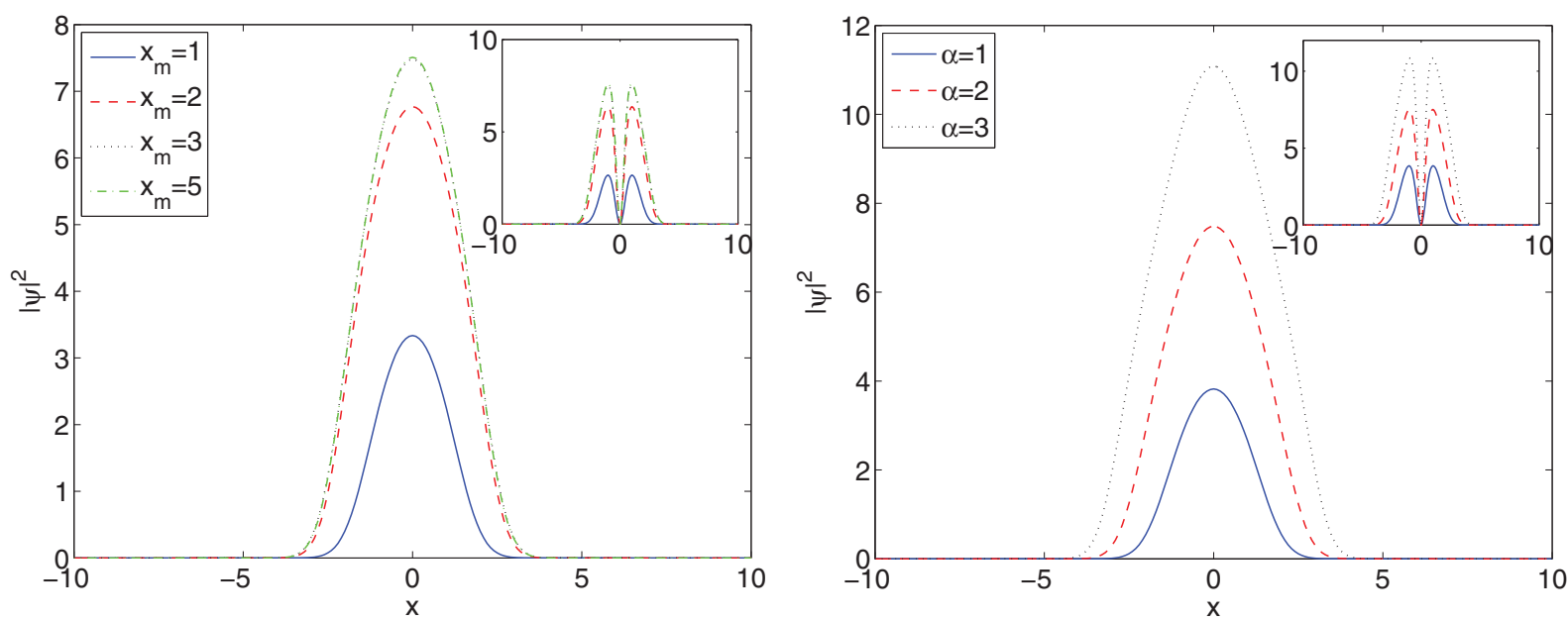

FIG. 1. (Color online) Spatial profiles of the densities $|\psi|^{2}$ of nodeless states and single dark solitons (insets). In the left (right) panel, the parameter $\alpha\left(x_{m}\right)$ is kept fixed, taking the value $\alpha=2\left(x_{m}=3\right)$, while $\sigma=3.5$ in both cases. As seen in the left panel, the profiles do not change appreciably above a critical value of $x_{m} \approx 3$, see, e.g., the profiles corresponding to $x_{m}=3$ [dotted (black) line] and $x_{m}=5$ [dotted-dashed (green) line], which are almost indistinguishable from each other. All quantities in this figure (and in all subsequent ones) are dimensionless. 
manifestation of stability inversions, not only can the nodeless state be stable while the dark soliton is not, but also vice versa: the dark-soliton state with a node can be stable while the state without a node is not. In Fig. 3 we show the details of the Bogolyubov spectra of both states. These showcase the dissipative nature of the dynamics being associated with frequencies chiefly with negative imaginary part; moreover, they also illustrate the potential instabilities arising in the system either in an oscillatory form through Hopf bifurcations associated with complex eigenfrequencies, or through zero crossings (and purely imaginary eigenfrequencies). This second scenario only appears for dark solitons. From an intuitive viewpoint, this phenomenology can partially be understood on the following grounds: the condensate in the absence of the external driving has an intrinsic length scale selected by the trap (and the chemical potential). The presence of the external forcing over the radius $x_{m}$ introduces an additional
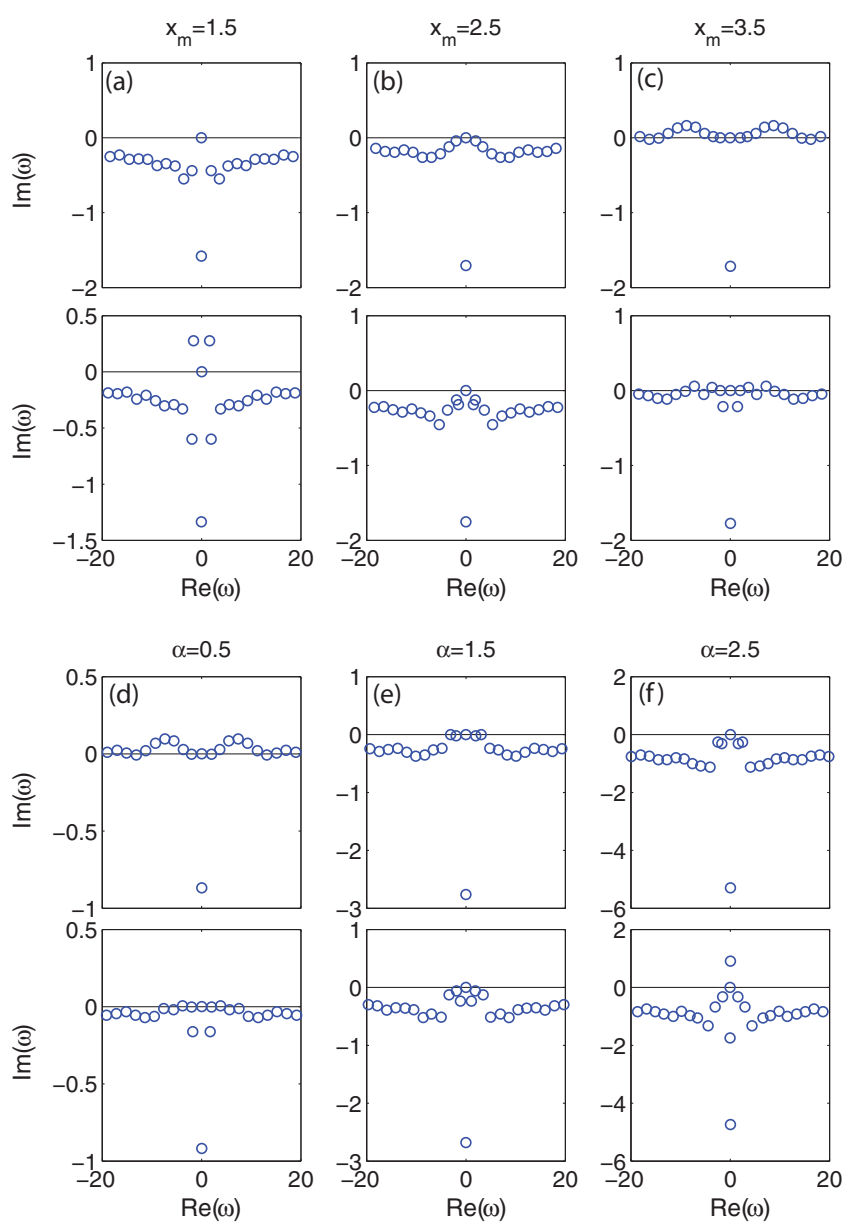

FIG. 3. (Color online) Spectral plane for nodeless clouds (first and third rows of panels) and dark solitons (second and fourth rows of panels) with $\sigma=0.35$. The top two rows of panels correspond to fixed $\alpha=1$ and increasing values of $x_{m}$, as labeled, whereas the bottom two rows of panels correspond to fixed $x_{m}=3$ and increasing values of $\alpha$, as indicated. The different cases correspond to the parameter locations depicted by the (green) circles in Fig. 2; cf. the stability or instability inferred by the absence or presence of eigenfrequencies with $\operatorname{Im}(\omega)>0$ in these spectra with the stability conclusions of Fig. 2.

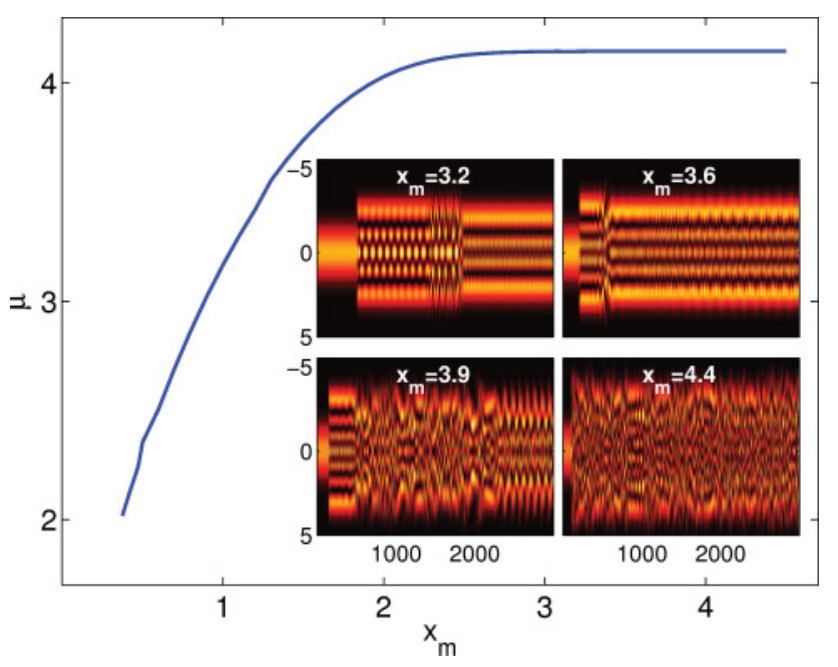

FIG. 4. (Color online) Chemical potential as a function of $x_{m}$ for a nodeless cloud with $\alpha=1.0$ and $\sigma=0.35$. The insets show the time evolution of the cloud for various values of $x_{m}$.

length scale competing with the former one. Hence, when this forcing becomes fairly (spatially) extended, it favors a spatially wider state. This is manifested through the instability of a group of "background" spectral modes (which are not the lowest modes of the condensate close to the spectral plane origin) in the panels of Fig. 3. On the contrary, the dark soliton or multi-soliton states may become unstable through the same mechanism, or they may also become unstable through their "internal modes" 22 that lead to the isolated instability through the zero crossing.

In Fig. 4 we show a continuation of the nodeless state for a fixed value of $\alpha=1.0$. In particular, we depict the chemical potential, $\mu$, as a function of the pumping-spot size, $x_{m}$. The insets show the dynamical evolution of the fixed-point solution for several illustrative values of $x_{m}$, which reflect the different possible dynamical phenomena. The values shown are in the unstable domain of the NC. There are two types of behavior.

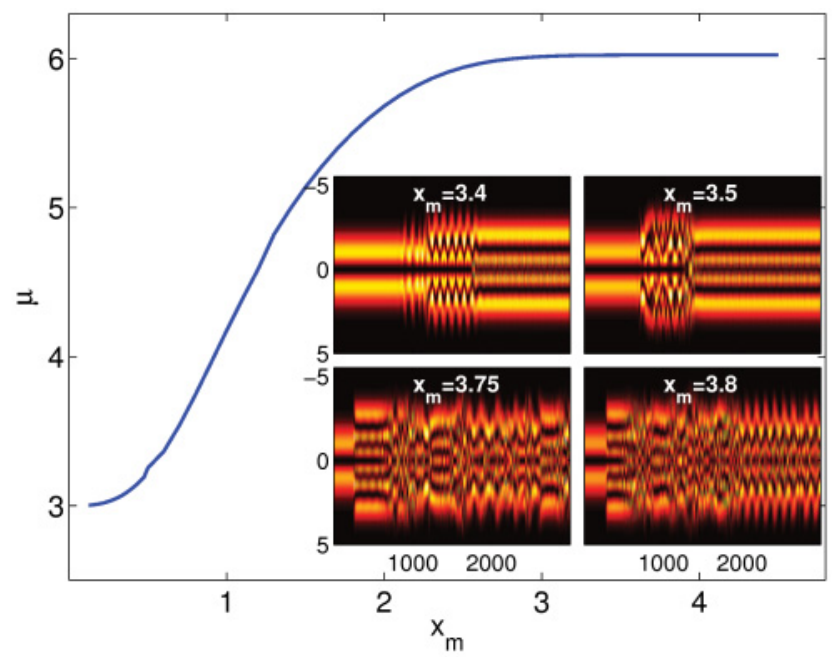

FIG. 5. (Color online) Chemical potential as a function of $x_{m}$ for a dark soliton with $\alpha=1.0$ and $\sigma=0.35$. The insets show the time evolution of the cloud for various values of $x_{m}$. 

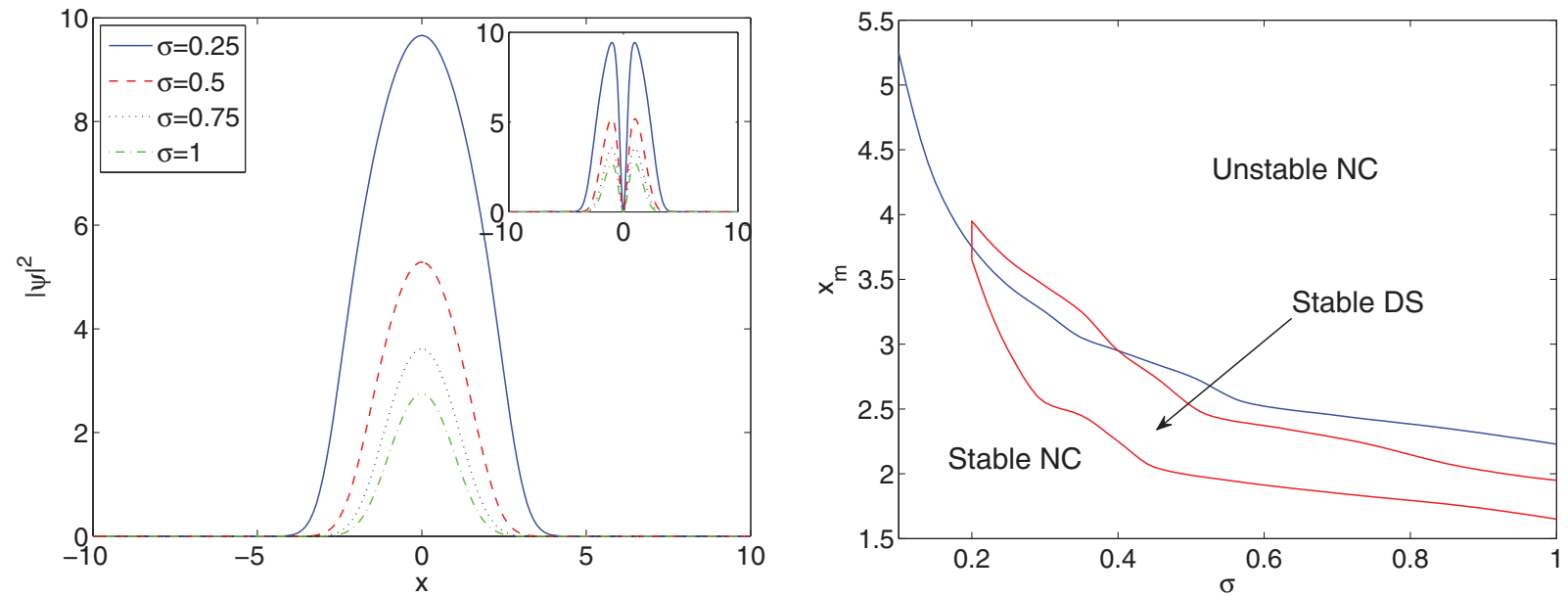

FIG. 6. (Color online) Left panel: Profiles for the nodeless clouds and dark solitons (inset) for different values of $\sigma$ and fixed $\alpha=2$ and $x_{m}=2.5$. Right panel: Stability domain for nodeless clouds (NC) and dark solitons (DS) for $\alpha=2$.

The first behavior, for $3.1<x_{m}<3.8$, corresponds to the NC decaying into multiple dark solitons, by means of oscillatory transients (even in the region of stability of the single DS). The spontaneous emergence of these states from a nodeless one is a feature particular to polariton BECs, having no analog in the atomic BEC case. On the other hand, a second behavior, corresponds to values $x_{m}>3.8$ : in this case, even though transient multi-soliton states still appear, they finally give rise to nearly "turbulent" nonlinear dynamics of a "sea" of multiple DS states, which may (or may not, depending on $x_{m}$ ) settle on an asymptotic multi-soliton state.

On the other hand, we have also investigated the dynamics of the fundamental (single) dark soliton in Fig. 5. We have found that, in their instability region, DSs decay toward the nodeless state, as expected, if the latter is stable. However, when the nodeless state is unstable, the dynamics is as follows: after a transient stage, a breathing multiple-DS structure is formed, which may consist of 3, 4, or 5 DSs. Examples of such evolutions are shown in the insets of Fig. 5. In this case, the DS is stable in the range $2.1<x_{m}<3.1$.
The effects of varying the parameters $\sigma$ and $x_{m}$ are depicted in Figs. 6 and 7 for fixed pumping-spot strengths $\alpha=2$ and $\alpha=3$, respectively. A new dynamical feature that arises for $\alpha=3$ and sufficiently large $\sigma$ and $x_{m}$ (see Fig. 7) is the spontaneous dynamical emergence of a highly asymmetric "sawtooth" structure (notice that for this value of $\alpha$, no stable DSs are found). Figure 8 shows how these sawtooth structures dynamically emerge from the NC when the latter is unstable. The left panel shows the emergence of a breathing sawtooth structure from an unstable NC. Notice that the asymmetry of this configuration is produced by the amplification of the asymmetric (small) perturbation added to the $\mathrm{NC}$ in order to manifest its dynamical instability. We have checked that this breathing behavior persists for very long times (larger than several thousands) without any apparent decay. This is due to the fact that, for these parameter values, the steady-state sawtooth configuration is unstable (see the spectrum depicted in the inset of the left panel in Fig. 8) and thus the cloud cannot decay to this state; rather it settles into an apparently stable breathing time-periodic sawtooth pattern. For other parameter
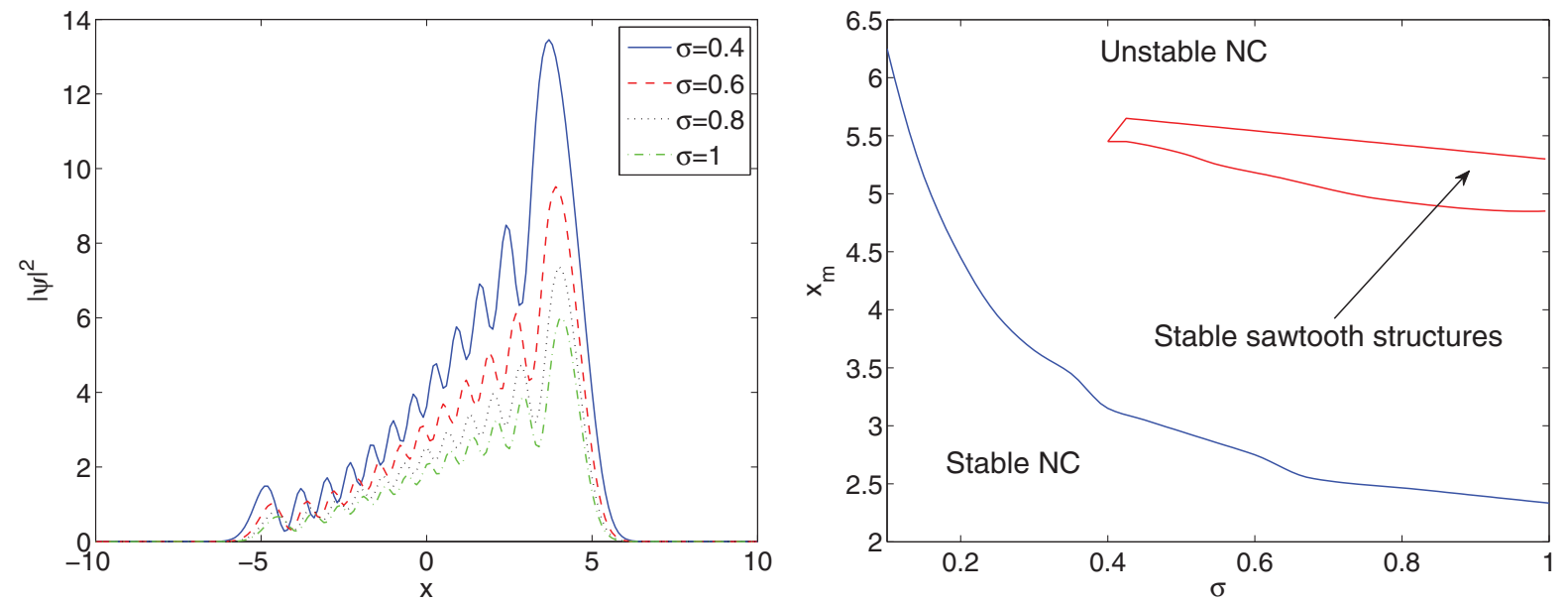

FIG. 7. (Color online) Left panel: Profiles of highly asymmetric "sawtooth" structures for different values of $\sigma$ and fixed $\alpha=3$ and $x_{m}=5$. Right panel: Stability domain for sawtooth structures with $\alpha=3$. 

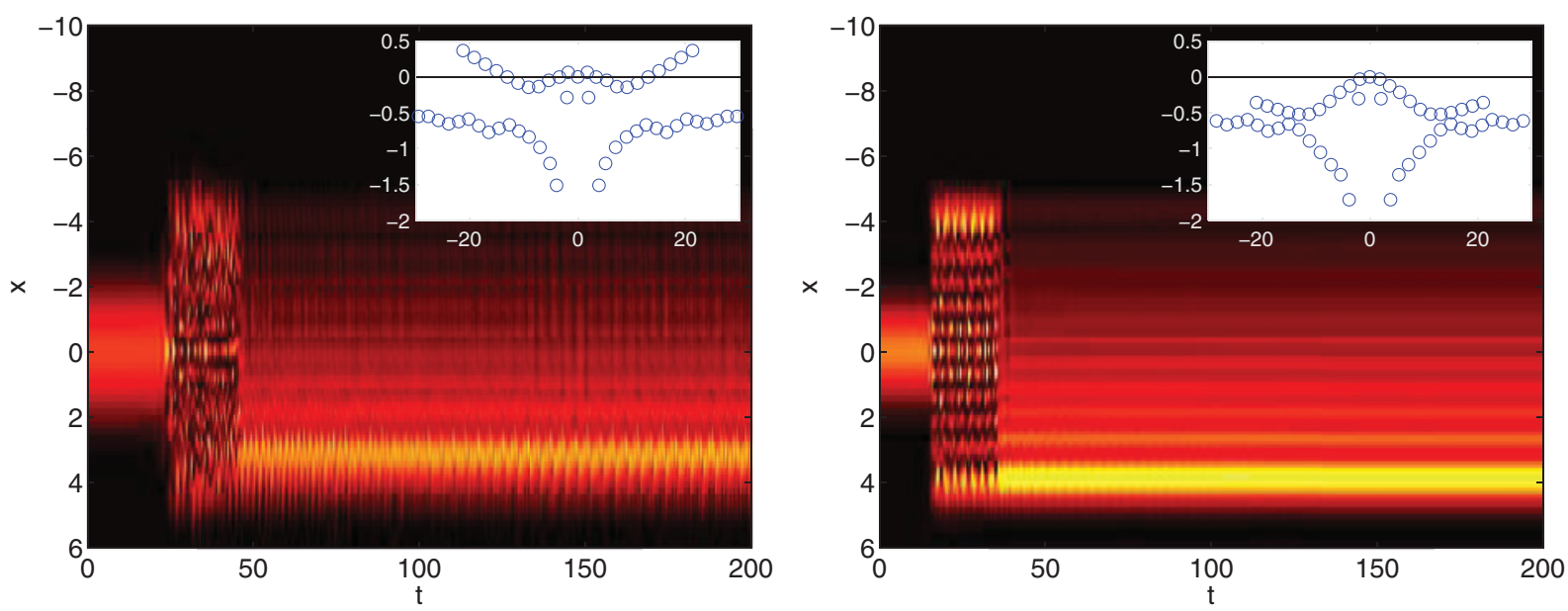

FIG. 8. (Color online) Density plots showing the evolution of unstable nodeless clouds to a breathing sawtooth structure for $\sigma=0.5$ (left) and to a nonbreathing (stable) sawtooth structure for $\sigma=1$ (right) with $x_{m}=5$. The inset in the left panel shows the linearization spectrum of the unstable sawtooth steady state about which the system oscillates for long times. The inset in the right panel corresponds to the linearization spectrum for the stable sawtooth steady state that the system asymptotes to.

values, the emerging sawtooth structures settle to stationary configurations without any breathing as can be seen seen in the right panel of Fig. 8. This case corresponds to parameter values inside the elongated island depicted in the right panel of Fig. 7 where these sawtooth configurations are stable.

The left panel of Fig. 7 depicts examples of stationary sawtooth structures. Similar to the saturation of the nodeless cloud size for large pumping-spot size $x_{m}$, we have also observed a saturation of the size for the sawtooth structures for large $x_{m}$ (results not shown here). It is extremely interesting that the polariton condensate is able to support stable asymmetric sawtooth-patterned states. Such solutions are not possible in atomic BECs, yet in the context of damped-driven systems, similar features have been recently observed in nonlinear

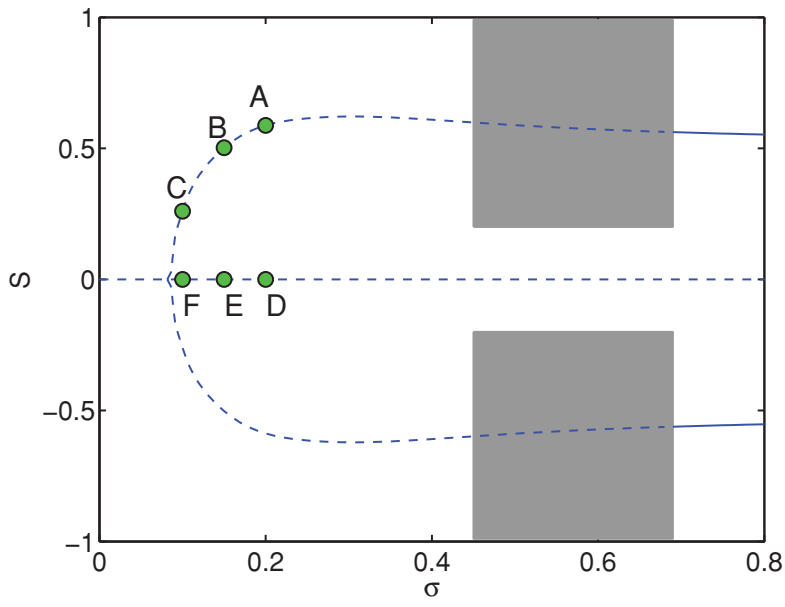

optical settings. ${ }^{34}$ Furthermore, it is important to mention that, because of the left-to-right symmetry of our system (around $x=0$ ), the sawtooth states appear in pairs (left- and right-handed sawtooth structures). Here, for simplicity, we only depict one family as the other one is a mirror image with respect to the center of the cloud. Such asymmetric solutions appear in our symmetric system through a symmetry-breaking pitchfork bifurcation scenario. In the typical supercritical pitchfork bifurcation scenario, as a system parameter is varied, a stable symmetric solution becomes unstable, while at the same time two stable asymmetric solutions (mirror images of each other) bifurcate beyond this critical point. ${ }^{35}$ However, in our case, there is a subtle difference, as the stationary bifurcating solutions are oscillatorily unstable.
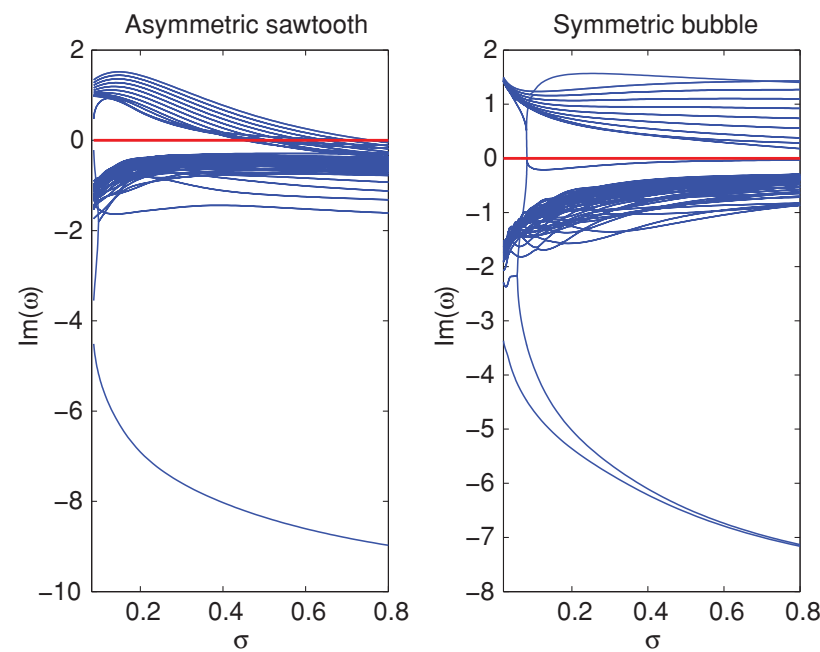

FIG. 9. (Color online) The left panel represents the symmetry parameter of Eq. (7) vs $\sigma$ for the symmetric bubble predecessor states and the asymmetric sawtooth offspring states resulting from the symmetry-breaking bifurcation. Dashed lines represent (oscillatory) unstable solutions whereas solid lines correspond to stable solutions. Shaded regions indicate the range where a Hopf bifurcation cascade takes place (notice that the symmetric solutions do not experience such a bifurcation). The (green) circles indicate the parameter locations for the solution profiles depicted in Fig. 10. The right panels show the evolution of the most relevant instability eigenvalues for both the bubble (right subpanel) and the sawtooth (left subpanel) solutions. The parameters $\alpha=3$ and $x_{m}=5$ are fixed. 

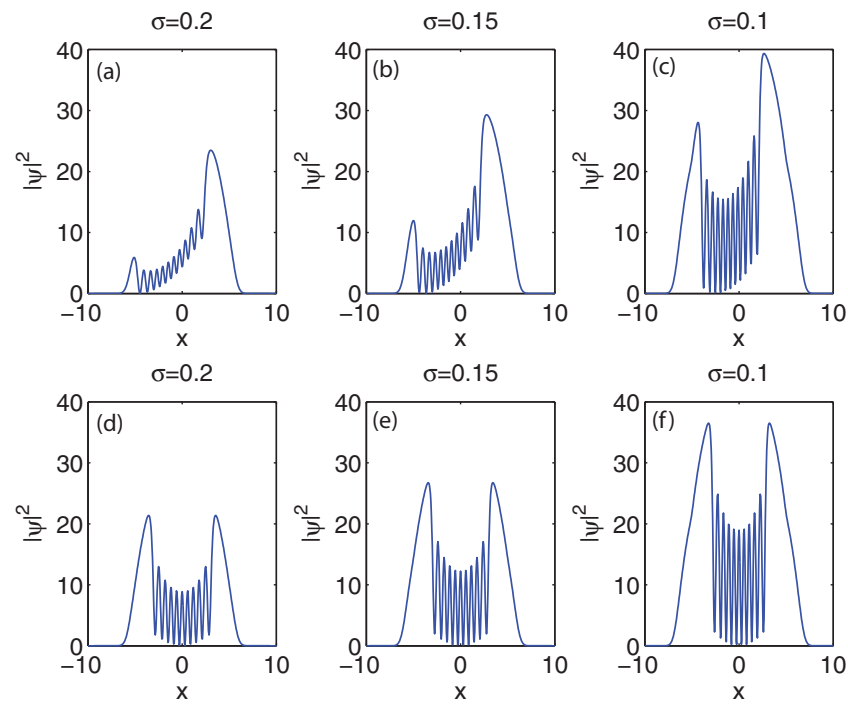

FIG. 10. (Color online) Profiles of sawtooth (top panels) and bubble solutions (bottom panels) for different values of $\sigma$ when $\alpha=3$ and $x_{m}=5$ are fixed. It is observed that the asymmetry of sawtooth solutions decreases as $\sigma$ decreases and, as expected, their profiles approach the symmetric bubble ones as the bifurcation point is approached. The different cases correspond to the parameter locations depicted by the (green) circles in the left panel of Fig. 9.

As can be seen in Figs. 9 and 10, where the symmetry parameter

$$
S=\frac{\int_{0}^{\infty} d x|\psi(x)|^{2}-\int_{-\infty}^{0} d x|\psi(x)|^{2}}{\int_{-\infty}^{\infty} d x|\psi(x)|^{2}}
$$

is depicted with respect to $\sigma$ for fixed $\alpha=3$ and $x_{m}=5$, the sawtooth structures bifurcate from symmetric bubble solutions at $\sigma=0.0817$. Such bubble states are depicted in the bottom panels of Fig. 10 (as corresponding to points D, E, and F within Fig. 9). These "ancestors" of the sawtooth patterns can be thought of as similar in intensity profile to the DS states, but without the phase jump. In fact, under appropriate conditions on the nonlinearity, such states exist but are unstable even in the GPE model in the absence of any trap. ${ }^{36}$ Their (oscillatory) instability in our setting is inherited by the ensuing sawtooth structures (while the bifurcation leads the bubbles to the acquisition of an additional purely imaginary eigenfrequency). Subsequently, however, as $\sigma$ increases, a cascade of inverse Hopf bifurcations progresses finally leading to the complete stabilization of the sawtooth states for $\sigma \geqslant 0.690$.

Finally, although our results already suggest that nonlinear excitations in the form of dark solitons should spontaneously emerge in polariton BECs, we offer some alternative dynamical schemes for producing such excitations, inspired by experimental realizations within their atomic BEC counterparts, ${ }^{22,31}$ which also appear to be within reach for the case of polaritons; see e.g., the very recent work of Ref. 21 and references therein. One possible nucleating mechanism for dark solitons is by dragging an obstacle-in the form of the potential of Eq. (6) - sufficiently fast through the condensate (see Refs. 31 and 21 for relevant experimental observations in atomic and polariton condensates, respectively). Examples of this effect are shown in Fig. 11. For the relatively strong harmonic confinement considered in Eq. (1), we have found that at most two DSs can survive due to their relatively large size. In this case, as shown in the left panel of Fig. 11, although three (or even four) DSs can be seen being emitted out of the defect path, through collisions they eventually decay down to two, which continue to interact. The DS nature of these structures can be seen through the phase jump of $\pi$ shown (together with its profile) in the inset. Chains of dark solitons (alias "dark-soliton trains") can be produced by this dragging-defect mechanism if one chooses a weaker harmonic trap $\Omega^{2} x^{2} / 2$ [instead of $x^{2}$ as in Eq. (1)], with a trap strength $\Omega$ sufficiently small. In fact, as depicted in the right panel of Fig. 11, a weaker trapping with $\Omega=0.04$ (corresponding to a considerably wider condensate) allows for the formation of a train of DSs that propagates initially in the opposite direction of the dragging defect. However, contrary to the Hamiltonian
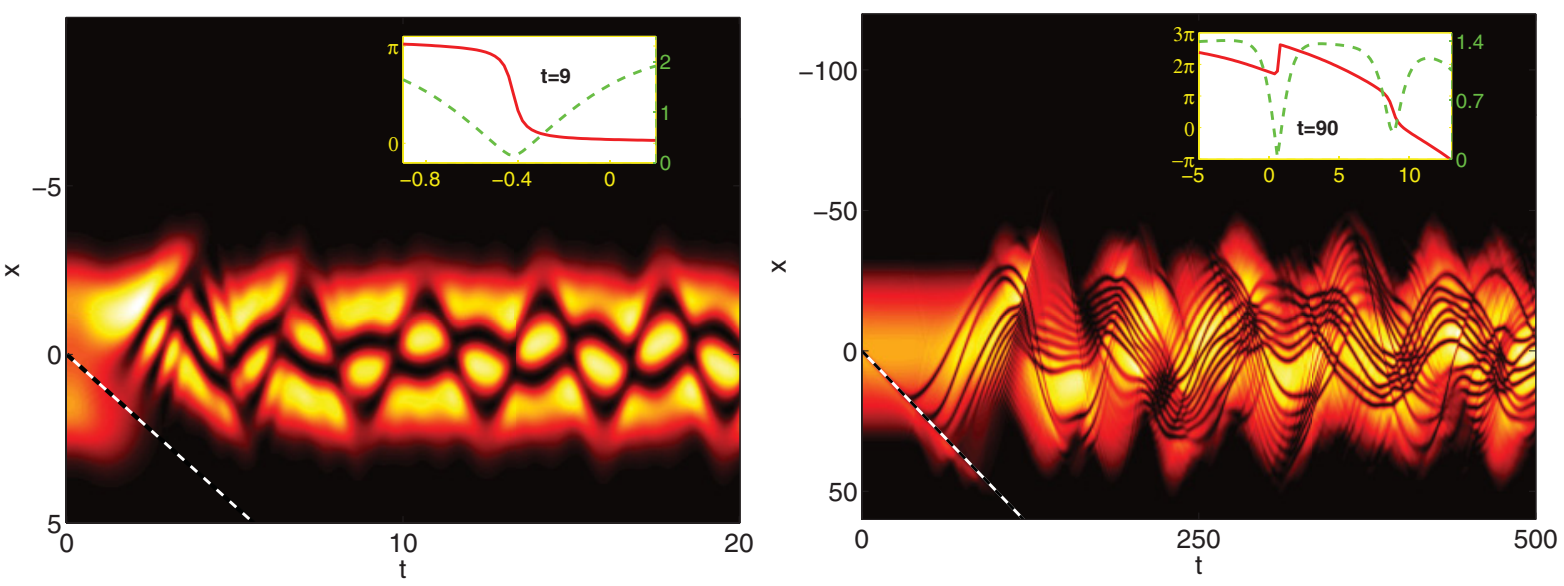

FIG. 11. (Color online) Nucleation of dark solitons by a dragging defect traversing a nodeless cloud. Depicted are the density plots of the nodeless cloud as it is traversed by the dragging defect. The dashed line represents the trajectory of the defect. The case depicted in the left panel corresponds to a tighter confinement with trap frequency $\Omega=1$, and parameter values $\alpha=1.0, \sigma=0.35$, and $x_{m}=2.8$, whereas $V_{0}=8, v=0.9$, and $\epsilon^{2}=4$. The right panel corresponds to a weaker harmonic trapping with trap frequency $\Omega=0.04$ and $\alpha=0.1, \sigma=0.08$, and $x_{m}=44.5$, while $V_{0}=1, v=0.5$, and $\epsilon^{2}=0.16$. The insets show the profile [dashed (green) line] and its corresponding phase [solid (red) line] at the indicated times. 

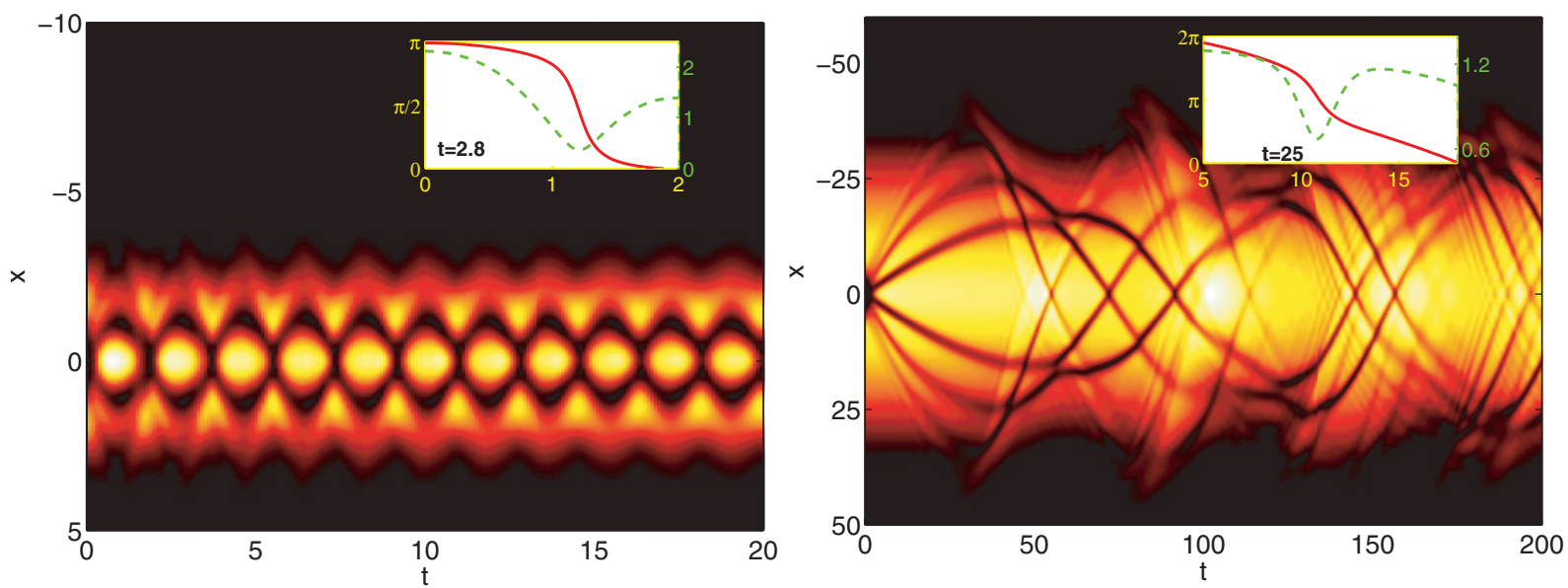

FIG. 12. (Color online) Nucleation of dark-soliton pairs by nonlinear interference of colliding condensate fragments. The plots depict the density of the evolution of a cloud created in a double well and allowed to interfere by removing the central barrier. Parameter values are (left) $\alpha=1.0, \sigma=0.35$, and $x_{m}=2.8$, whereas $V_{0}=17, \epsilon^{2}=1$, and $\Omega=1$; (right) $\alpha=0.1, \sigma=0.08$, and $x_{m}=44.5$, whereas $V_{0}=4$ and $\epsilon^{2}=16$, while the trap frequency is $\Omega=0.04$. The insets show the profile [dashed (green) line] and its corresponding phase [solid (red) line] at the indicated times.

case of atomic BECs where the distance between the generated DSs appears to be approximately constant, ${ }^{37}$ here, DSs interact strongly with each other and with the background cloud, setting it in oscillation. This might also be due to the unstable nature of the underlying NC for this parameter set. Again the DS nature is visible from the $\pi$ phase jump at each of the nonlinear excitations shown in the inset.

Another possible nucleation mechanism for DSs is by nonlinear interference (see e.g., Ref. 27 for a discussion in the context of atomic BECs). This mechanism can be realized by splitting the condensate into two fragments by adiabatically inducing a potential barrier in the central portion of a stationary $\mathrm{NC}$, and subsequently releasing the fragments by suddenly removing the barrier that separates them. This "nonlinearinterference method" was used in atomic BEC experiments to demonstrate the generation of vortex structures ${ }^{38}$ (see also the theoretical work in Ref. 39) and dark solitons. ${ }^{22,29,40}$ Figure 12 depicts two samples of this nucleation mechanism where two polariton condensates, initially placed in a double-well potential, are released in the harmonic trap and allowed to interfere. Again we show the scenarios of strong (left) and weak(er) harmonic confinement (right). We considered an initial situation where a barrier of the same shape as (but wider than) the obstacle considered before [cf. Eq. (6)] is superposed at the center of the harmonic trap. At $t=0$ the barrier is removed, thus allowing the two portions of the condensate to mix and interfere. As can be seen in the left panel of Fig. 12, a pair of dark pulses is formed; but contrary to the case of the soliton trains nucleated by the dragging defect, we observe that the DSs nucleated by the nonlinear-interference mechanism move relatively fast away from each other toward the edge of the condensate. Notice that, as again shown in the inset, the phase jumps of approximately $\pi$ indicate that these are pairs of genuine DSs. The right panel shows the case of weaker confinement where in this case more than one pair of nonlinear excitations is produced by the nonlinear interference.

\section{CONCLUSIONS}

In this work, we considered and studied a complex Gross-Pitaevskii model describing the quasi-one-dimensional dynamics of polariton condensates. Our motivation was to understand the fundamental differences between polariton condensates and their atomic (Hamiltonian BECs) counterparts. We found that the specific pumping and damping terms that have been argued as being relevant to the polariton condensates case offer a wide range of unexpected features when compared to atomic condensates.

The fundamental nodeless state (regarded as the "ground state") of the system was found to become unstable through a variety of mechanisms, while excited states-in the form of single or multiple dark solitons-were found to result from the instability of the nodeless state. The fundamental excited state, namely the single dark soliton, was also found to be subject to instabilities, leading to either spontaneous formation of multidark-soliton states or even to emergence of a "dark-soliton turbulence" (when highly unstable). All these are dynamical manifestations that significantly distinguish the polaritonic case from the atomic BEC variant of the problem, yet we have intuitively attributed them to the emerging competition of length scales (among the intrinsic length scale of the trapped system and the length scale of the applied forcing). We also observed the emergence of stable asymmetric sawtooth-like configurations which are not present in atomic BECs and arise from symmetry-breaking bifurcations of symmetric unstable bubble states.

Finally, other techniques, namely nucleation of dark solitons by dragging an obstacle through the condensate and through nonlinear interference of colliding condensate fragments, have been studied. These were adapted from the atomic-condensate case in order to produce fundamental nonlinear excitations - in the form of dark solitons. It was shown that both techniques were efficient in doing so. 
There are many interesting avenues that this work opens in the way of future directions. On the one hand, it would be useful to try to develop analytical tools to understand the instability of dark solitons in polariton condensates, as well as that of the backgrounds (i.e., the nodeless clouds) on top of which these "live". On the other hand, it would be interesting to extend some of the present considerations-such as the spectral analysis of stationary states, the nonlinear interference technique, or the polariton flow around static impurities ${ }^{41}$ for producing coherent structures in the $2 \mathrm{D}$ case-to examine some of the theoretical and potentially even experimentally relevant (in the latter case) results thereof. Another interesting aspect that is worth investigating is the comparison between the 1D statics and dynamics of attractive polariton condensates $^{42}$ and their atomic BEC counterparts. Such studies are currently in progress and will be reported in future publications.

\section{ACKNOWLEDGMENTS}

J.C. acknowledges financial support from the MICINN project FIS2008-04848. A.S.R. gratefully acknowledges the hospitality from the Department of Mathematics of the University of Massachusetts, financial support from FCT through grant SFRH/BSAB/1035/2010, and the use of computational resources from GOE-Inesc Porto. R.C.G. gratefully acknowledges the hospitality of the Grupo de Física No Lineal (GFNL, University of Sevilla, Spain) and support from NSF-DMS-0806762, Plan Propio de la Universidad de Sevilla, Grant No. IAC09-I-4669 of Junta de Andalucia and Ministerio de Ciencia e Innovación, Spain. P.G.K. acknowledges the support from NSF-DMS-0806762 and from the Alexander von Humboldt Foundation. The work of D.J.F. was partially supported by the Special Account for Research Grants of the University of Athens.
${ }^{1}$ J. Kasprzak, M. Richard, S. Kundermann, A. Baas, P. Jeambrun, J. M. J. Keeling, F. M. Marchetti, M. H. Szymańska, R. André, J. L. Staehli, V. Savona, P. B. Littlewood, B. Deveaud, and L. S. Dang, Nature (London) 443, 409 (2006); R. Balili, V. Hartwell, D. Snoke, L. Pfeiffer, and K. West, Science 316, 1007 (2007); W. Lai, N. Y. Kim, S. Utsunomiya, G. Roumpos, H. Deng, M. D. Fraser, T. Byrnes, P. Recher, N. Kumada, T. Fujisawa, and Y. Yamamoto, Nature (London) 450, 529 (2007); H. Deng, G. S. Solomon, R. Hey, K. H. Ploog, and Y. Yamamoto, Phys. Rev. Lett. 99, 126403 (2007).

${ }^{2}$ C. J. Pethick and H. Smith, Bose-Einstein Condensation in Dilute Gases (Cambridge University Press, Cambridge, 2002); L. P. Pitaevskii and S. Stringari, Bose-Einstein Condensation (Oxford University Press, Oxford, 2003).

${ }^{3}$ G. Björk, S. Machida, Y. Yamamoto, and K. Igeta, Phys. Rev. A 44, 669 (1991); C. Weisbuch, M. Nishioka, A. Ishikawa, and Y. Arakawa, Phys. Rev. Lett. 69, 3314 (1992).

${ }^{4}$ The Physics of Semiconductor Microcavities, edited by B. Deveaud (Wiley-VCH, Weinheim, 2007).

${ }^{5}$ M. Richard, J. Kasprzak, A. Baas, S. Kundermann, K. G. Lagoudakis, M. Wouters, I. Carusotto, R. André, B. DeveaudPledran, and L. S. Dang, Int. J. Nanotech. 7, 668 (2010).

${ }^{6}$ A. Amo, J. Lefrère, S. Pigeon, C. Adrados, C. Ciuti, I. Carusotto, R. Houdré, E. Giacobino, and A. Bramati, Nat. Phys. 5, 805 (2009).

${ }^{7}$ K. G. Lagoudakis, M. Wouters, M. Richard, A. Baas, I. Carusotto, R. André, L. S. Dang, and B. Deveaud-Plédran, Nat. Phys. 4, 706 (2008).

${ }^{8}$ M. D. Fraser, G. Roumpos, and Y. Yamamoto, New J. Phys. 11, 113048 (2009).

${ }^{9}$ A. Amo, D. Sanvitto, F. P. Laussy, D. Ballarini, E. del Valle, M. D. Martin, A. Lemaître, J. Bloch, D. N. Krizhanovskii, M. S. Skolnick, C. Tejedor, and L. Viña, Nature (London) 457, 291 (2009).

${ }^{10}$ A. Amo, T. C. H. Liew, C. Adrados, R. Houdré, E. Giacobino, A. V. Kavokin, and A. Bramati, Nat. Photonics 4, 361 (2010).

${ }^{11}$ S. I. Tsintzos, N. T. Pelekanos, G. Konstantinidis, Z. Hatzopoulos, and P. G. Savvidis, Nature (London) 453, 372 (2008).

${ }^{12}$ J. Keeling and N. G. Berloff, Phys. Rev. Lett. 100, 250401 (2008).
${ }^{13}$ M. O. Borgh, J. Keeling, and N. G. Berloff, Phys. Rev. B 81, 235302 (2010).

${ }^{14}$ P. G. Kevrekidis, D. J. Frantzeskakis, and R. Carretero-González, Emergent Nonlinear Phenomena in Bose-Einstein Condensates: Theory and Experiment (Springer-Verlag, Heidelberg, 2008).

${ }^{15}$ M. Wouters and I. Carusotto, Phys. Rev. Lett. 99, 140402 (2007).

${ }^{16}$ M. Wouters, I. Carusotto, and C. Ciuti, Phys. Rev. B 77, 115340 (2008).

${ }^{17}$ C. Ciuti and I. Carusotto, Phys. Status Solidi B 242, 2224 (2005).

${ }^{18}$ H. Deng, H. Haug, and Y. Yamamoto, Rev. Mod. Phys. 82, 1489 (2010).

${ }^{19}$ R. I. Kaitouni, O. El Daï, A. Baas, M. Richard, T. Paraïso, P. Lugan, T. Guillet, F. Morier-Genoud, J. D. Ganière, J. L. Staehli, V. Savona, and B. Deveaud, Phys. Rev. B 74, 155311 (2006); O. El Daï, A. Baas, T. Guillet, J.-P. Brantut, R. I. Kaitouni, J. L. Staehli, F. Morier-Genoud, and B. Deveaud, Appl. Phys. Lett. 88, 061105 (2006); D. Bajoni, E. Peter, P. Senellart, J. L. Smirr, I. Sagnes, A. Lemaître, and J. Bloch, ibid. 90, 051107 (2007); D. Bajoni, P. Senellart, E. Wertz, I. Sagnes, A. Miard, A. Lemaître, and J. Bloch, Phys. Rev. Lett. 100, 047401 (2008); R. Cerna, D. Sarchi, T. K. Paraïso, G. Nardin, Y. Léger, M. Richard, B. Pietka, O. El Daïf, F. Morier-Genoud, V. Savona, M. T. Portella Oberli, and B. Deveaud-Plédran, Phys. Rev. B 80, 121309(R) (2009); M. Wouters and T. C. H. Liew, and V. Savona, ibid. 82, 245315 (2010).

${ }^{20}$ E. Wertz, L. Ferrier, D. D. Solnyshkov, R. Johne, D. Sanvitto, A. Lemaître, I. Sagnes, R. Grousson, A. V. Kavokin, P. Senellart, G. Malpuech, and J. Bloch, Nat. Phys. 6, 860 (2010).

${ }^{21}$ A. Amo, S. Pigeon, D. Sanvitto, V. G. Sala, R. Hivet, I. Carusotto, F. Pisanello, G. Leménager, R. Houdré, E. Giacobino, C. Ciuti, and A. Bramati, Science 32, 1167 (2011).

${ }^{22}$ A. Weller, J. P. Ronzheimer, C. Gross, J. Esteve, M. K. Oberthaler, D. J. Frantzeskakis, G. Theocharis, and P. G. Kevrekidis, Phys. Rev. Lett. 101, 130401 (2008); G. Theocharis, A. Weller, J. P. Ronzheimer, C. Gross, M. K. Oberthaler, P. G. Kevrekidis, and D. J. Frantzeskakis, Phys. Rev. A 81, 063604 (2010).

${ }^{23}$ R. I. Kaitouni, O. El Daïf, A. Baas, M. Richard, T. Paraïso, P. Lugan, T. Guillet, F. Morier-Genoud, J. D. Ganière, J. L. Staehli, 
V. Savona, and B. Deveaud, Phys. Rev. B 74, 155311 (2006); O. El Daif, A. Baas, T. Guillet, J.-P. Brantut, R. I. Kaitouni, J. L. Staehli, F. Morier-Genoud, and B. Deveaud, Appl. Phys. Lett. 88, 061105 (2006).

${ }^{24}$ R. Balili, D. W. Snoke, L. Pfeiffer, and K. West, Appl. Phys. Lett. 88, 031110 (2006); R. Balili, V. Hartwell, D. Snoke, L. Pfeiffer, and K. West, Science 316, 1007 (2007); V. Negoita, D. W. Snoke, and K. Eberl, Appl. Phys. Lett. 75, 2059 (1999).

${ }^{25}$ C.-K. Lam, B. A. Malomed, K. W. Chow, and P. K. A. Wai, Eur. Phys. J. Special Topics 173, 233 (2009); C. H. Tsang, B. A. Malomed, C.-K. Lam, and K. W. Chow, Eur. Phys. J. D 59, 81 (2010); F. Kh. Abdullaev, V. V. Konotop, M. Salerno, and A. V. Yulin, Phys. Rev. E 82, 056606 (2010); V. Skarka, N. B. Aleksic', H. Leblond, B. A. Malomed, and D. Mihalache, Phys. Rev. Lett. 105, 213901 (2010); M. J. Ablowitz, T. P. Horikis, S. D. Nixon, and D. J. Frantzeskakis, Opt. Lett. 36, 793 (2011); M. J. Ablowitz, S. D. Nixon, T. P. Horikis, and D. J. Frantzeskakis, e-print arXiv:1008.3756 (unpublished); Proc. Roy. Soc. London A (in press).

${ }^{26}$ B. A. Malomed, O. Dzyapko, V. E. Demidov, and S. O. Demokritov, Phys. Rev. B 81, 024418 (2010).

${ }^{27}$ D. J. Frantzeskakis, J. Phys. A 43, 213001 (2010).

${ }^{28}$ C. Becker, S. Stellmer, P. Soltan-Panahi, S. Dörscher, M. Baumert, E.-M. Richter, J. Kronjäger, K. Bongs, and K. Sengstock, Nat. Phys. 4, 496 (2008); S. Stellmer, C. Becker, P. Soltan-Panahi, E.-M. Richter, S. Dörscher, M. Baumert, J. Kronjäger, K. Bongs, and K. Sengstock, Phys. Rev. Lett. 101, 120406 (2008).

${ }^{29}$ I. Shomroni, E. Lahoud, S. Levy, and J. Steinhauer, Nat. Phys. 5, 193 (2009).
${ }^{30}$ Z. Dutton, M. Budde, C. Slowe, and L. V. Hau, Science 293, 663 (2001).

${ }^{31}$ P. Engels and C. Atherton, Phys. Rev. Lett. 99, 160405 (2007).

${ }^{32}$ E. Cancellieri, F. M. Marchetti, M. H. Szymańska, and C. Tejedor, Phys. Rev. B 82, 224512 (2010)

${ }^{33}$ M. Wouters and I. Carusotto, Phys. Rev. Lett. 105, 020602 (2010).

${ }^{34}$ Y. V. Kartashov, V. V. Konotop, and V. A. Vysloukh, Phys. Rev. A 83, 041806(R) (2011); Opt. Lett. 36, 82 (2011).

${ }^{35}$ S. H. Strogatz, Nonlinear Dynamics and Chaos (Perseus Books, Reading, MA, 1994).

${ }^{36}$ A. de Bouard, SIAM J. Math. Anal. 26, 566 (1995).

${ }^{37}$ R. Carretero-González, P. G. Kevrekidis, D. J. Frantzeskakis, B. A. Malomed, S. Nandi, and A. R. Bishop, Math. Comput. Simul. 74, 361 (2007).

${ }^{38}$ D. R. Scherer, C. N. Weiler, T. W. Neely, and B. P. Anderson, Phys. Rev. Lett. 98, 110402 (2007).

${ }^{39}$ R. Carretero-González, N. Whitaker, P. G. Kevrekidis, and D. J. Frantzeskakis, Phys. Rev. A 77, 023605 (2008); R. CarreteroGonzález, B. P. Anderson, P. G. Kevrekidis, D. J. Frantzeskakis, and C. N. Weiler, ibid. 77, 033625 (2008).

${ }^{40}$ J. J. Chang, P. Engels, and M. A. Hoefer, Phys. Rev. Lett. 101, 170404 (2008).

${ }^{41}$ S. Pigeon, I. Carusotto, and C. Ciuti, Phys. Rev. B 83, 144513 (2011).

${ }^{42}$ O. A. Egorov, D. V. Skryabin, A. V. Yulin, and F. Lederer, Phys. Rev. Lett. 102, 153904 (2009). 